\title{
Human tuberculosis caused by Mycobacterium bovis: a retrospective comparison with Mycobacterium tuberculosis in a Mexican tertiary care centre, 2000-2015
}

Pedro Torres-Gonzalez', Miguel E. Cervera-Hernandez', Areli Martinez-Gamboa', Lourdes Garcia-Garcia², Luis P. Cruz-Hervert ${ }^{2}$, Miriam Bobadilla-del Valle ${ }^{1}$, Alfredo Ponce-de Leon ${ }^{1}$ and Jose Sifuentes-Osornio ${ }^{3^{*}}$

\begin{abstract}
Background: Human tuberculosis caused by Mycobacterium bovis is believed to be frequent in developing countries. Transmission is usually through ingestion of unpasteurized dairy products, although airborne contagion is possible. Disease caused by M. tuberculosis or M. bovis is clinically indistinguishable from each other. The aim of this study was to determine the factors associated with M. bovis disease.

Methods: Retrospective analysis of all culture-positive cases of M. bovis and M. tuberculosis from 2000 to 2015, in a Mexican tertiary-care centre. Sociodemographic, clinical, and radiographic data from medical records were compared. Disease site was classified as pulmonary, extrapulmonary, or pulmonary and extrapulmonary, based on cultures.

Results: We evaluated 533 cases, 372 (69.7\%) of which were caused by M. tuberculosis and 161 (30.2 \%) by M. bovis. Characteristics associated with $M$. bovis disease were: younger age (aOR 0.97, $95 \% \mathrm{Cl} 0.95-0.98)$, glucocorticoid use (aOR 2.27, $95 \% \mathrm{Cl} 1.42-3.63$ ), and extrapulmonary disease (aOR 1.80, $95 \% \mathrm{Cl} 1.21-2.69$ ). M. tuberculosis was associated with lower socioeconomic status (aOR 0.52, $95 \% \mathrm{Cl}$ 0.28-0.97). When we analysed only pulmonary cases, younger age (aOR 0.97, 95 \% Cl 0.96-0.99), glucocorticoid use (aOR 2.41, 95 \% Cl 1.30-4.46), and smoking (aOR 1.94, Cl $95 \%$ 1.15-3.27) were associated with M. bovis. Both groups showed similar proportions of direct microscopy smear results (respiratory samples) and chest $X$-ray cavitations.
\end{abstract}

Conclusions: Younger age, glucocorticoid use, and extrapulmonary disease were associated with $M$. bovis as the causative agent of tuberculosis in a group of patients from a tertiary care centre in a country where bovine tuberculosis is endemic. Further studies must be conducted in the general population to determine pathogen-specific associated factors and outcomes.

Keywords: Tuberculosis, Mycobacterium bovis, Mexico, Epidemiology, Clinical characteristics

\footnotetext{
* Correspondence: sifuentesosornio@gmail.com

${ }^{3}$ Department of Medicine, Instituto Nacional de Ciencias Médicas y Nutrición

Salvador Zubirán, Av. Vasco de Quiroga \#15, Tlalpan, Belisario Domínguez

Sección XVI, 14080 Mexico City, Mexico

Full list of author information is available at the end of the article
} 


\section{Background}

Mycobacterium bovis, a member of the Mycobacterium tuberculosis complex, causes tuberculosis (TB) in cattle and a wide range of mammals including humans, although to a lesser extent than Mycobacterium tuberculosis. The implementation of bovine TB (BTB) eradication programs and universal pasteurization of dairy products in developed countries has reduced the disease in these regions, and thus clinicians often overlook the possibility of human TB caused by $M$. bovis $[1,2]$. Conversely, in developing countries, $M$. bovis disease is likely to be still frequent but underreported because of reliance on laboratory techniques that are insufficient to identify the different species within the $M$. tuberculosis complex (i.e., direct smear microscopy, GeneXpert molecular testing, or culture without species-level identification) [3].

Disease caused by either M. bovis or M. tuberculosis is considered clinically and radiologically indistinguishable from each other [4]. Nevertheless, M. bovis is naturally resistant to pyrazinamide, an essential component of the first-line anti-TB drug regimen [5]. Hence, prompt and correct identification of the causative agent can have important implications for patient outcomes [6].

The main route of transmission is through consumption of unpasteurized dairy products, and thus M. bovis usually presents as extrapulmonary disease [1]. Before genetic investigations were common, human-to-human spread via aerosolized particles was a subject of controversy [7]. However, recent reports from hospital and community outbreaks indicate that this type of transmission is feasible [8,9]. Still, irrespective of its route of transmission, pulmonary disease accounts for a sizeable proportion of all $M$. bovis cases in developed countries, and perhaps much more in endemic regions [10].

Recent reports underscore the importance of understanding the epidemiology of $M$. bovis disease $[11,12]$. Younger age, HIV coinfection, and other immunosuppressive conditions are some of the described associated factors [11]. Of note, a connection with Mexico (in the form of country of birth, contact with family members, or consumption of unpasteurized dairy products) has been the most frequently reported risk factor in research conducted in the United States $[6,10,11,13-15]$. In this context, the aim of this study was to describe the sociodemographic, epidemiological, and clinical characteristics, and to determine the factors associated with $M$. bovis disease as compared with M. tuberculosis.

\section{Methods}

\section{Setting and participants}

This study was conducted at a 230-bed, national referral centre for adult patients with complex medical and surgical problems, located in Mexico City with approximately 6000 discharges annually. This centre's laboratory of clinical microbiology maintains a database containing information on all positive mycobacterial cultures. We searched this database to identify all patients with a positive culture for $M$. tuberculosis or $M$. bovis who were admitted to the hospital during the period 2000-2015.

\section{Variables and definitions}

We reviewed the medical records of the identified patients and retrieved variables of interest using a specially designed case report form. Patients with incomplete or missing medical records were excluded from the study. Socioeconomic status was evaluated based on the hospital's 7-point score assigned by a social worker upon admission. This scale is based on family income, occupation, educational level, number of house residents, number of school-age children in the family, construction material of the house, ownership of current residence, setting (urban or rural), and availability of services (electricity, paved roads, running water, and sewage) [16]. We defined low socioeconomic status as a grade $<2$. Also, we categorized patients as residents of a non-accredited zone for BTB eradication by the United States Department of Agriculture using data provided by the Servicio Nacional de Sanidad Inocuidad y Calidad Agroalimentaria (SENASICA). This is the government office in charge of monitoring BTB control in Mexico [17]. Pulmonary disease was defined as a positive culture from sputum, bronchoalveolar lavage, endotracheal aspirate, gastric aspirate, lung and pleural biopsy, pleural fluid, or intrathoracic lymph nodes. Extrapulmonary disease was defined as a positive culture from any other anatomical site. Glucocorticoid use was defined as $\geq 15 \mathrm{mg}$ of prednisone or its equivalent for more than one month in the year previous to TB diagnosis [18]. Multidrug resistance (MDR) was defined as resistance to at least both rifampicin and isoniazid.

\section{Microbiology procedures}

Samples for mycobacteria identification were digested, decontaminated by the NALC- $\mathrm{NaOH}$ method $[19,20]$, and inoculated in both Löwenstein-Jensen medium and MGIT tubes (Becton-Dickinson, Sparks, MA), according to the manufacturer's specifications. Samples labelled as biopsies were additionally inoculated in Stonebrink culture medium. All positive cultures were further identified by DNA probe (Accuprobe, San Diego, CA). During the study period, all $M$. tuberculosis complex isolates were identified to the species level. We defined $M$. bovis as a positive culture with dysgonic growth and biochemical tests results (niacin production, nitrate reduction, thiophen-2-carboxylic acid anhydride susceptibility, and pyrazinamidase deamidation) typical of this species, and M. tuberculosis as a positive culture with eugonic growth and compatible biochemical test results (niacin production, 
nitrate reductase positive test and heat-stable catalase activity) [21]. Additionally, both $M$. bovis and M. tuberculosis identification was confirmed by spoligotyping from 2000 to 2013 and by the GenoType MTBC (Hain Lifescience $\mathrm{GmbH}$, Nehren, Germany) test from 2013 to 2015 [22, 23]. Susceptibility testing for rifampicin, streptomycin, isoniazid, and ethambutol was performed using the radiometric BACTEC 460 or the BACTEC 960 culture system (Becton-Dickinson, Sparks, MA) [24].

\section{Statistical methods}

The characteristics of $M$. bovis and M. tuberculosis cases were compared by univariate analysis using the $\chi^{2}$ test, Fisher's exact test, Student's unpaired $t$-test, and the Mann-Whitney $U$ test, as appropriate. To determine characteristics associated with $M$. bovis as compared with $M$. tuberculosis we used unconditional logistic regression. The models were validated by evaluating their suitability, model specificity and multicollinearity. Variables included in the models were those with $p$-values $<0.2$ in the univariate analysis or with biological plausibility. The final model was achieved using a hierarchical backward elimination approach. The variable association was expressed as adjusted odds ratio $(\mathrm{aOR})$ at $95 \%$ confidence interval (CI). All statistical analyses were performed using STATA 11.0 software (StataCorp, College Station, TX).

\section{Results}

We identified 610 patients with culture-positive TB identified to the species level for the period 2000-2015. We excluded 77 (61 M. tuberculosis, 16 M. bovis) because of missing or incomplete records. Of the remaining 533 cases, 372 (69.7\%) were identified as $M$. tuberculosis and 161 (30.2 \%) as M. bovis. Twenty-nine percent $(155 / 533)$ of these cases were diagnosed from 2000 to $2004,27.4 \%(146 / 533)$ from 2005 to 2009 and $43.5 \%(232 / 533)$ from 2010 to 2015 . The proportion of $M$. bovis isolates from each period were $24.5 \%$ (38/155), $32.8 \%(48 / 146)$ and $32.3 \%$ (75/232), respectively.

\section{Sociodemographic characteristics and comorbidities}

Fifty-six percent $(303 / 533)$ of the cases were male and $22.8 \%(122 / 533)$ occurred in presence of HIV co-infection. The univariate analysis indicated that younger age, connective tissue disease, and glucocorticoid use were more frequent among patients with $M$. bovis disease. Conversely, patients with $M$. tuberculosis presented diabetes mellitus diagnosis more frequently. History of consumption of unpasteurized dairy products was often missing from clinical records as it was reported in 5/15 (33.3\%) of the $M$. bovis cases and 10/27 (37\%) of M. tuberculosis $(p=0.81)$.
Neither residency in a non-accredited zone for BTB eradication, nor positive HIV status were significantly different among $M$. bovis cases (Table 1).

\section{Clinical and microbiological characteristics}

Among the 533 patients, MDR was detected in the first cultured isolates of seven patients. Six of these were $M$. tuberculosis (4 pulmonary, 2 extrapulmonary), and one was $M$. bovis (pulmonary). The univariate analysis showed differences between the proportions of $M$. bovis and $M$. tuberculosis cases concerning disease site, presenting symptoms, and mean tuberculin skin test (TST) induration. Extrapulmonary disease accounted for $41.6 \%$ of M. bovis cases, and $30.1 \%$ of M. tuberculosis $(p=0.01)$. Pulmonary disease was observed in $36 \%$ of M. bovis, and $52.6 \%$ of $M$. tuberculosis $(p<0.001)$. Limiting the analysis to patients with extrapulmonary disease only, $M$. bovis presented with abdominal TB in $32.8 \%$ of the cases, compared with $10.7 \%$ for M. tuberculosis $(p<0.001)$. No differences were observed with respect to disease site when limiting the analysis to patients with both pulmonary and extrapulmonary disease. Regarding clinical presentation, $M$. bovis patients more frequently reported fever and gastrointestinal symptoms, whereas $M$. tuberculosis cases reported more respiratory symptoms. The mean TST induration among $M$. bovis cases was $9.6 \mathrm{~mm}$, compared with $14 \mathrm{~mm}$ in M. tuberculosis cases $(p=0.02$; Table 2). We observed no difference between both species regarding inhospital, 90-day mortality (20/161; $12.4 \%$ M. bovis vs. 51/372; $13.7 \%$ M. tuberculosis).

\section{Characteristics of pulmonary disease}

When we restricted the analysis to cases with pulmonary involvement, younger age, smoking, glucocorticoid use, and normal and miliary chest X-ray (CXR) appearance were associated with $M$. bovis. In contrast, working in a farm, low socioeconomic status, presenting with cough, and nodule on CXR were more frequent among $M$. tuberculosis cases. Direct microscopy smear results of respiratory samples were similar for both groups (Table 3).

\section{Multivariate analysis}

In the overall analysis, younger age (aOR 0.97; $95 \%$ CI 0.95-0.98), glucocorticoid use (aOR 2.27; $13495 \%$ CI 1.42-3.63), extrapulmonary disease (aOR 1.80; CI 1.21-2.69) and higher socioeconomic status (aOR 0.52; $95 \%$ CI 0.28-0.97) were independently associated with $M$. bovis disease. When we restricted the analysis to pulmonary cases, younger age (aOR 0.97; $95 \%$ CI 0.96-0.99), glucocorticoid use (aOR 2.41; $95 \%$ CI 1.30-4.46), and smoking (aOR 1.94; 95 \% CI 1.15-3.27) were independently associated with $M$. bovis (Table 4). 
Table 1 Sociodemographic characteristics and comorbidities of patients with tuberculosis caused by Mycobacterium bovis or Mycobacterium tuberculosis, Mexico City, 2000-2015

\begin{tabular}{|c|c|c|c|}
\hline \multirow[b]{2}{*}{ Characteristic } & \multicolumn{2}{|c|}{ Etiological agent } & \multirow[b]{2}{*}{$p^{\mathrm{a}}$} \\
\hline & $\begin{array}{l}\text { M. bovis } \\
(n=161)\end{array}$ & $\begin{array}{l}\text { M. tuberculosis } \\
(n=372)\end{array}$ & \\
\hline Age, years, median (IQR) & $38(29-52)$ & $49(33-64)$ & $<0.001^{b}$ \\
\hline Sex (female) & $66 / 161(40.9)$ & $164 / 372(44)$ & 0.5 \\
\hline Healthcare worker & $5 / 151(3.3)$ & $19 / 360(5.2)$ & 0.33 \\
\hline Farm worker & $5 / 151(3.3)$ & $26 / 360(7.2)$ & 0.09 \\
\hline Low socioeconomic status & $17 / 161(10.5)$ & $62 / 372(16.6)$ & 0.068 \\
\hline BCG-vaccinated & $82 / 118(69.4)$ & $179 / 261(68.5)$ & 0.86 \\
\hline Contact with TB-infected people & 18/139 (12.9) & $67 / 335(20)$ & 0.069 \\
\hline Resides in a non-accredited zone for bovine-TB eradication ${ }^{c}$ & 140/159 (88) & $303 / 370(81.8)$ & 0.078 \\
\hline Alcohol intake $\geq 40 \mathrm{~g} /$ day & $9 / 152(5.9)$ & $36 / 354(10.1)$ & 0.12 \\
\hline Smoker (present) & $71 / 153(46.4)$ & $147 / 356(41.2)$ & 0.28 \\
\hline HIV coinfection & $43 / 161(26.7)$ & $79 / 372(21.2)$ & 0.16 \\
\hline CD4 count $\leq 200$ cells $/ \mathrm{mm}^{3}$ & $31 / 35(88.5)$ & $60 / 67(89.5)$ & 0.87 \\
\hline Diabetes mellitus & 23/161 (14.2) & $84 / 372(22.5)$ & 0.028 \\
\hline Connective tissue disease $^{d}$ & 29/161 (18) & $43 / 372(11.5)$ & 0.045 \\
\hline Solid organ transplant recipient & 4/161 (2.4) & $8 / 372(2.1)$ & 0.81 \\
\hline Charlson comorbidity index $\geq 3$ & 72/161 (44.7) & $150 / 372(40.3)$ & 0.34 \\
\hline Previous TB & $2 / 161(1.2)$ & $14 / 372(3.7)$ & 0.11 \\
\hline Glucocorticoid use & $46 / 161(28.5)$ & $54 / 372(14.5)$ & $<0.001$ \\
\hline
\end{tabular}

Data are $\mathrm{n} / \mathrm{N}$ (\%) of cases, unless otherwise indicated. $n$ values across categories might be less than the counts in the column headings due to missing data $I Q R$ Interquartile range, TB Tuberculosis, HIV Human immunodeficiency virus, BCG Bacillus Calmette-Guérin

${ }^{a} p$ values were determined by the $x^{2}$ test

${ }^{\mathrm{b}} p$ value was determined by Student's $t$-test

'As determined by SENASICA

${ }^{d}$ Systemic lupus erythematosus, rheumatoid arthritis, Sjögren syndrome, dermatomyositis, ankylosing spondylitis, mixed connective tissue disease, systemic sclerosis

${ }^{e} \geq 15 \mathrm{mg} / \mathrm{d}$ of prednisone or equivalent dose of steroids for 1 month or more in the year prior to TB diagnosis

\section{Discussion}

In this study, conducted in a country considered of sporadic occurrence of BTB, we observed sociodemographic, clinical, and radiographic differences between $M$. bovis and $M$. tuberculosis cases. Younger age, glucocorticoid use, and extrapulmonary disease were associated with $M$. bovis. Cigarette smoking was additionally associated with $M$. bovis among the pulmonary cases. M. tuberculosis was associated with lower socioeconomic status.

We found a higher proportion of $M$. bovis in comparison to similar hospital-based studies. We previously reported this fact in a study encompassing local and referred samples from other hospitals in the region $[3,25,26]$. Also, we reported an uprising tendency in the proportion of M. bovis isolates through time. This may be attributed to the larger study period, and a higher proportion of immunosuppressed population along with the poor control of BTB in the region, especially among dairy farms and artisan dairy products retail $[3,27]$.

In this study, $M$. bovis cases were significantly younger; this was true for all clinical presentations even after adjustment with comorbidities that may present in the younger population such as connective tissue diseases or hematologic malignancies. Contrary to what others have suggested, this may indicate a shorter latency period for $M$. bovis disease $[12,28]$. However, it is also possible that unpasteurized dairy consumption may be more frequent at younger ages; unfortunately, this variable was often missing from the clinical record, and thus it was impossible for us to ascertain this fact. Studies accurately exploring the time of exposure and unpasteurized dairy consumption are needed in order to confirm these hypotheses.

A large proportion of our study population was receiving glucocorticoids in the year previous to TB diagnosis; this is explained by the fact that our hospital treats a large population of patients with autoimmune diseases. Glucocorticoids modify the cellular response, a critical component for the containment of TB infection, and have been previously described as a risk factor for disease reactivation $[29,30]$. In this study, glucocorticoids were associated with $M$. bovis, suggesting that immunosuppression may favour M. bovis disease. In fact, tuberculin skin 
Table 2 Clinical and microbiologic characteristics of patients with tuberculosis caused by Mycobacterium bovis or Mycobacterium tuberculosis, Mexico City, 2000-2015

\begin{tabular}{|c|c|c|c|}
\hline \multirow[b]{2}{*}{ Characteristic } & \multicolumn{2}{|c|}{ Etiological agent } & \multirow[b]{2}{*}{$p^{\mathrm{a}}$} \\
\hline & $\begin{array}{l}\text { M. bovis } \\
(n=161)\end{array}$ & $\begin{array}{l}\text { M. tuberculosis } \\
(n=372)\end{array}$ & \\
\hline \multicolumn{4}{|l|}{ Anatomical site of isolation } \\
\hline Pulmonary & $58 / 161(36)$ & 196/372 (52.6) & $<0.001$ \\
\hline Extrapulmonary & $67 / 161(41.6)$ & $112 / 372(30.1)$ & 0.01 \\
\hline Pulmonary and Extrapulmonary & $36 / 161(22.3)$ & $64 / 372(17.2)$ & 0.16 \\
\hline \multicolumn{4}{|c|}{ Primary site of isolation (Restricted to pulmonary cases with and extrapulmonary involvements cases ${ }^{b}$ ) } \\
\hline Abdominal $^{c}$ & $7 / 36(19.4)$ & 22/64 (34.3) & 0.11 \\
\hline Genitourinary & $16 / 36(44.4)$ & 22/64 (34.3) & 0.31 \\
\hline Central Nervous System & 10/36 (27.7) & $9 / 64(14)$ & 0.093 \\
\hline Lymph node & $0 / 36(0)$ & $1 / 64(1.5)$ & - \\
\hline Skin, joint, bone, and soft tissue & $3 / 36(8.3)$ & $2 / 64(3.1)$ & 0.25 \\
\hline Blood, bone marrow, liver, and spleen & $0 / 36(0)$ & $8 / 64(12.5)$ & - \\
\hline \multicolumn{4}{|c|}{ Primary site of isolation (Restricted to extrapulmonary cases $^{d}$ ) } \\
\hline Abdominal $^{c}$ & $22 / 67(32.8)$ & 12/112 (10.7) & $<0.001$ \\
\hline Genitourinary & $9 / 67(13.4)$ & 26/112 (23.2) & 0.11 \\
\hline Central Nervous System & 14/67 (20.9) & 17/112 (15.1) & 0.32 \\
\hline Lymph node & $15 / 67(22.3)$ & $37 / 112(33)$ & 0.12 \\
\hline Skin, joint, bone, and soft tissue & $4 / 67(5.9)$ & $13 / 112(11.6)$ & 0.21 \\
\hline Blood, bone marrow, liver, and spleen & $3 / 67(4.4)$ & $7 / 112(6.2)$ & 0.61 \\
\hline \multicolumn{4}{|c|}{ Primary site of isolation (Restricted to pulmonary cases with and extrapulmonary involvement cases ${ }^{d}$ ) } \\
\hline Abdominal $^{c}$ & $7 / 36(19.4)$ & 22/64 (34.3) & 0.11 \\
\hline Genitourinary & $16 / 36(44.4)$ & $22 / 64(34.3)$ & 0.31 \\
\hline Central Nervous System & 10/36 (27.7) & 9/64 (14) & 0.093 \\
\hline Lymph node & $0 / 36(0)$ & $1 / 64(1.5)$ & - \\
\hline Skin, joint, bone, and soft tissue & $3 / 36(8.3)$ & $2 / 64(3.1)$ & 0.25 \\
\hline Blood, bone marrow, liver, and spleen & $0 / 36(0)$ & $8 / 64(12.5)$ & - \\
\hline Symptom onset to diagnosis, days, median (IQR) & $63(32-154)$ & $90(34-197)$ & $0.089^{e}$ \\
\hline \multicolumn{4}{|l|}{ Presenting symptoms } \\
\hline Fever & 127/159 (79.8) & $254 / 364(69.7)$ & 0.019 \\
\hline Weight loss & $72 / 159(45.2)$ & 181/364 (49.7) & 0.38 \\
\hline Cough & $60 / 159(37.7)$ & 195/364 (53.5) & 0.001 \\
\hline Haemoptysis & $5 / 159(3.1)$ & 29/364 (7.9) & 0.039 \\
\hline Gastrointestinal $^{f}$ & $71 / 159(44.6)$ & $117 / 364(32.3)$ & 0.007 \\
\hline Neurological & $30 / 159(18.8)$ & 63/364 (17.3) & 0.67 \\
\hline Genitourinary & 10/159 (6.2) & 29/364 (7.9) & 0.49 \\
\hline Primary multidrug resistance ${ }^{g}$ & $1 / 161(0.6)$ & $6 / 372(1.6)$ & 0.35 \\
\hline Mean TST induration, mm, (IQR) & $9.6(0-18)$ & $14(0-25)$ & $0.02^{\mathrm{e}}$ \\
\hline$\geq 5 \mathrm{~mm}$ & $30 / 76(39.4)$ & $91 / 172(52.9)$ & 0.051 \\
\hline$\geq 10 \mathrm{~mm}$ & 29/76 (38.1) & $91 / 172(52.9)$ & 0.032 \\
\hline$\geq 15 \mathrm{~mm}$ & 23/76 (30.2) & 79/172 (45.9) & 0.021 \\
\hline
\end{tabular}

Data are $\mathrm{n} / \mathrm{N}$ (\%) of cases, unless otherwise indicated. $n$ values across categories might be less than the counts in the column headings due to missing data IQR Interquartile range, TST tuberculin skin test

${ }^{a} p$ values were determined by the two-sample test of proportion or the $x^{2}$ test, as appropriate

${ }^{\mathrm{b}}$ Restricted to cases with extrapulmonary disease only

'Gastrointestinal, peritoneal, and faecal samples

${ }^{\mathrm{d}}$ Restricted to cases with both pulmonary and extrapulmonary disease

${ }^{e} p$ value was determined by the Mann-Whitney $U$ test

Diarrhoea, vomiting, and abdominal pain

${ }^{9}$ Resistance to at least both isoniazid and rifampicin 
Table 3 Clinical and radiographic characteristics of patients with pulmonary ${ }^{a}$ tuberculosis caused by Mycobacterium bovis or Mycobacterium tuberculosis, Mexico City, 2000-2015

\begin{tabular}{|c|c|c|c|}
\hline \multirow[b]{2}{*}{ Characteristic } & \multicolumn{2}{|c|}{ Etiological agent } & \multirow[b]{2}{*}{$p^{b}$} \\
\hline & $\begin{array}{l}\text { M. bovis } \\
(n=94)\end{array}$ & $\begin{array}{l}\text { M. tuberculosis } \\
(n=260)\end{array}$ & \\
\hline Age, years, median (IQR) & $39(30-51)$ & $46(30-64)$ & $0.0053^{c}$ \\
\hline Sex (female) & 33/94 (35.1) & $99 / 260(38)$ & 0.61 \\
\hline Healthcare worker & $3 / 88(3.4)$ & $15 / 255(5.8)$ & 0.37 \\
\hline Farm worker & 2/88 (2.2) & 22/255 (8.6) & 0.044 \\
\hline Low socioeconomic status & 9/94 (9.5) & $49 / 260(18.8)$ & 0.037 \\
\hline BCG-vaccinated & $42 / 63(66.6)$ & 130/186 (69.8) & 0.63 \\
\hline Contact with TB-infected people & $11 / 80(13.7)$ & $48 / 235(20.4)$ & 0.18 \\
\hline Resides in high-prevalence region of bovine $\mathrm{TB}^{\mathrm{d}}$ & 83/93 (89.2) & 209/259 (80.6) & 0.06 \\
\hline Alcohol intake $\geq 40 \mathrm{~g} /$ dayay & $5 / 89(5.6)$ & $30 / 251(11.9)$ & 0.09 \\
\hline Unpasteurized dairy products consumption & 2/10 (20) & $8 / 17(47)$ & 0.16 \\
\hline Smoker (present) & $50 / 89(56.1)$ & $103 / 253(40.7)$ & 0.012 \\
\hline HIV coinfection & $26 / 94(27.6)$ & $63 / 260(24.2)$ & 0.51 \\
\hline CD4 count $\leq 200$ cells $/ \mathrm{mm}^{3}$ & $20 / 21(95.2)$ & $46 / 51(90.2)$ & 0.48 \\
\hline Diabetes mellitus & 20/94 (21.2) & $60 / 260(23)$ & 0.72 \\
\hline Connective tissue disease $^{e}$ & 17/94 (18) & $28 / 260(10.7)$ & 0.068 \\
\hline Charlson comorbidity index $\geq 3$ & 46/94 (48.9) & $121 / 260(46.5)$ & 0.69 \\
\hline Glucocorticoid use $\mathrm{f}^{\mathrm{f}}$ & 25/94 (26.6) & $37 / 260(14.2)$ & 0.007 \\
\hline Symptom onset to diagnosis, days, median (IQR) & $62(35-131)$ & $80(33-182)$ & $0.15^{9}$ \\
\hline \multicolumn{4}{|l|}{ Presenting symptoms } \\
\hline Fever & 75/93 (80.6) & $194 / 255(76)$ & 0.36 \\
\hline Weight loss & 48/93 (51.6) & $137 / 255(53.7)$ & 0.72 \\
\hline Cough & $47 / 93(50.5)$ & $171 / 255(67)$ & 0.005 \\
\hline Haemoptysis & $5 / 93(5.3)$ & $26 / 255(10.2)$ & 0.16 \\
\hline Dyspnoea & 25/93 (26.8) & $81 / 255(31.7)$ & 0.38 \\
\hline \multicolumn{4}{|l|}{ Chest radiography pattern } \\
\hline Normal & 13/79 (16.4) & 19/242 (7.8) & 0.027 \\
\hline Cavitary & $9 / 79(11.3)$ & $33 / 242(13.6)$ & 0.6 \\
\hline Miliary & $30 / 79(37.9)$ & $63 / 242(26)$ & 0.042 \\
\hline Pleural effusion & $17 / 79(21.5)$ & $40 / 242(16.5)$ & 0.31 \\
\hline Consolidation & $13 / 79(16.4)$ & $65 / 242(26.8)$ & 0.061 \\
\hline Nodule & $1 / 79(1.2)$ & 23/242 (9.5) & $0.013^{h}$ \\
\hline Interstitial & $1 / 79(1.2)$ & $10 / 242(4.1)$ & $0.3^{h}$ \\
\hline Respiratory sample positive smear & $39 / 88(44.3)$ & $132 / 252(52.3)$ & 0.19 \\
\hline
\end{tabular}

Data are $\mathrm{n} / \mathrm{N}$ (\%) of cases, unless otherwise indicated. $n$ values across categories might be less than the counts in the column headings due to missing data IQR Interquartile range, BCG Bacillus Calmette-Guérin, TB Tuberculosis, HIV Human immunodeficiency virus

aEcludes cases that involved extrapulmonary disease only

${ }^{\mathrm{b}} p$ values were determined by the $x^{2}$ test

${ }^{c} p$ value was determined by Student's $t$-test

${ }^{\mathrm{d}}$ As determined by SENASICA

eSystemic lupus erythematosus, rheumatoid arthritis, Sjögren syndrome, dermatomyositis, ankylosing spondylitis, mixed connective tissue disease, systemic sclerosis

${ }^{f} \geq 15 \mathrm{mg} / \mathrm{d}$ of prednisone or equivalent dose of steroids for 1 month or more in the year prior to TB diagnosis

${ }^{g} p$ value was determined by the Mann-Whitney $U$ test

${ }^{h} p$ values were determined by Fisher's exact test 
Table 4 Multivariate analysis of factors associated with Mycobacterium bovis compared with Mycobacterium tuberculosis, Mexico City, 2000-2015

\begin{tabular}{lll}
\hline & All cases & Pulmonary cases $^{\mathrm{a}}$ \\
Factor & Adjusted OR (95 \% Cl) & Adjusted OR (95 \% Cl) \\
\hline Age & $0.97(0.95-0.98)$ & $0.97(0.96-0.99)$ \\
Resides in non-accredited zone for bovine-TB eradication ${ }^{\text {b }}$ & $1.68(0.94-3.0)$ & $1.67(0.77-3.62)$ \\
Low socioeconomic status & $0.52(0.28-0.97)$ & $0.48(0.21-1.1)$ \\
Glucocorticoid use & $2.27(1.42-3.63)$ & $2.41(1.30-4.46)$ \\
Extrapulmonary disease $_{\text {Smoker (present) }}$ & $1.80(1.21-2.69)$ & $1.61(0.93-2.80)$ \\
\hline
\end{tabular}

Values were determined by logistic regression

$O R$ Odds ratio, $C l$ Confidence interval, TB Tuberculosis

${ }^{a}$ Excludes cases that involved extrapulmonary disease only

${ }^{b}$ As determined by SENASICA

${ }^{c} \geq 15 \mathrm{mg} / \mathrm{d}$ of prednisone or equivalent dose of steroids for $1 \mathrm{month}$ or more in the year before TB diagnosis

test reactivity was also significantly lower amongst $M$. bovis-infected patients, supporting a higher degree of immunosuppression. The proportion of cases co-infected with HIV included in this report was higher that the 2014 WHO reports for Mexico (9\%) [31]. However, we did not identify an association between HIV coinfection and $M$. bovis disease, even after stratification by CD4+ counts. Similar studies from non-endemic regions have reported contradictory results or borderline associations with HIV $[10,11,15]$. This finding suggests that, in a high $M$. bovisburden country, the risk of TB caused by either species is similar among people living with HIV.

The prevalence of M. bovis in humans correlates with that of BTB. This has been established by the decrease in prevalence after the implementation of BTB prevention programs in Europe [2]. Reports from developed countries show that most cases occur among immigrants. In fact, the majority of the studies conducted in the United States have identified a connection with Mexico [11, 15]. We and others have previously described that close contact with cattle is an associated factor for developing $M$. bovis disease [32]; in the present study, we tried to measure this effect by identifying patients who lived in non-accredited regions for BTB eradication, but we were unable to find a significant association. This might be explained by the geographical location of our hospital and the fact that most of our patients reside in the central states of Mexico, where the highest prevalence of BTB is reported, and because exposure-related data such as unpasteurized dairy consumption and close contact with cattle were missing from most of the clinical records [17]. Additionally, changes in the definition of high-prevalence zones regarding BTB have occurred through time due new requirements for cattle export; therefore, we were unable to compare this variable through the study period and this may also have accounted for the lack of association. We observed a higher proportion of $M$. tuberculosis among patients with a lower socioeconomic status. This may be related to conditions such as living in crowded settings, alcoholism, and malnutrition, which may be more critical for an exclusively airborne pathogen like M. tuberculosis [33].

Traditionally, $M$. bovis has been associated with extrapulmonary disease; however, it is unclear if this is related to route of contagion, the immune status of the host, or if it obeys to particular virulence features of M. bovis $[2,14,34]$. In this study, the proportion of extrapulmonary disease was higher than the 2014 WHO reports for Mexico $(\approx 20 \%)$, and we found a similar proportion of patients with concurrent pulmonary and extrapulmonary disease among both species, but a higher proportion of $M$. bovis causing exclusively extrapulmonary disease, suggesting that extrapulmonary spread is not determined by species but may be determined by the route of contagion.

We report a sizeable amount of pulmonary M. bovis, which is consistent with previous reports $[10,11,15]$. The proportion of patients that reported contact with TB-infected people was similar among both groups. This suggests that human-to-human airborne transmission of $M$. bovis may possibly contribute to the TB burden. In fact, a previous study found a similar rate of tuberculin skin test positivity among household contacts of patients with $M$. bovis and M. tuberculosis [7]. However, we do not have information regarding the causing species among the contacts and this precludes us from reaching a conclusion. Also, it is possible that contacts may share other sources of contagion such as contact with cattle, unpasteurized dairy products, and exposure to other TB cases in an intermediate burden setting. We also found smoking as an associated factor for $M$. bovis among pulmonary cases. Previously, it has been reported as a risk factor for TB because smoking causes local alterations of the lung immune response and dysfunctional mucociliary clearance [35-38]. In the case of a less airborne- 
efficient pathogen such as $M$. bovis, these alterations may be more relevant.

In the same way as in previous studies, we found similar proportions of positive direct microscopy smears and cavitations in the CXR [7, 14]. These are considered the most important features that determine the contagiousness in pulmonary TB. Therefore, we believe that pulmonary $M$. bovis patients must be subject to the same isolation measures as $M$. tuberculosis in order to avoid spread. With respect to other radiographic patterns in the CXR, patients with a positive culture for $M$. bovis were more frequently reported without abnormalities. This may indicate the need to perform more detailed imaging studies whenever $M$. bovis is suspected.

We did not observe a difference regarding in-hospital mortality between both species, even after stratification by disease site. However, we did not perform a detailed mortality analysis due to the lack of severity of disease variables necessary for adjustment. Furthermore, it is also likely that in-hospital mortality may have been overestimated in our study due to the overrepresentation of immunosuppressed patients. Regarding the impact of anti-tuberculosis treatment, it may be difficult to assess the impact of the absence of the pyrazinamide effect on $M$. bovis during the initial phase of treatment when other drugs are administered concomitantly. However, we believe that species identification is more critical for relapse and treatment failure rates in the long-term, given that the continuation phase of treatment may need to be prolonged (as with any pyrazinamide resistant isolate) [39]. This may be overlooked by clinicians in a setting where susceptibility testing and/or species identification is not performed. Unfortunately, currently we are not able to analyse long-term outcomes. Virtually all patients are only seen in our hospital during the acute phase of the disease, and are later followed by their local health clinic.

This study has some limitations. The retrospective design precluded us from exploring important variables that are subject to the quality of the original interview, including known risk factors for $M$. bovis infection such as consumption of unpasteurized dairy products and contact with cattle. Also, the search for additional extrapulmonary sites was not performed systematically, but rather it was symptom oriented, and perhaps the isolation of $M$. bovis may have triggered a more exhaustive search. Additionally, we did not include data from children in this study given that our hospital only tends to the adult population, precluding us from further exploring this age group as a risk factor. This is important because TB caused by $M$. bovis has been described more frequently among children [40]. Finally, the patient population of this tertiary care centre may not be representative of the rest of the country. We have a large cohort of immunosuppressed people, and the majority of the patients reside in the central region of the country where BTB is more prevalent. Therefore, studies performed in the general population are needed to further elucidate the role of BTB prevalence as risk factor for M. bovis in humans.

\section{Conclusions}

In conclusion, younger age, glucocorticoid use, and extrapulmonary disease were associated with $M$. bovis disease among patients of a tertiary care hospital, whereas low socioeconomic status was associated with $M$. tuberculosis disease in this same group. These differences demonstrate that, although considered very closely related mycobacteria species with indistinguishable clinical presentations, clinicians must be aware of the possibility of $M$. bovis in the presence of these features in similar settings, especially because it may be required to adjust treatment length in the absence of pyrazinamide activity. However, further studies in the general population with a proper follow-up are needed to generalize these findings and define other associated factors for $M$. bovis disease.

\section{Abbreviations}

aOR: Adjusted odds ratio; BTB: Bovine tuberculosis; Cl: Confidence interval; CXR: Chest X-ray; MDR: Multidrug resistance; SENASICA: Servicio Nacional de Sanidad Inocuidad y Calidad Agroalimentaria; TB: Tuberculosis;

TST: Tuberculin skin test

\section{Acknowledgments}

We thank Andres Traslosheros Enkerlin, Eva Plasencia Moran, Florentina Medina Arellano, and Hugo Enriquez Sanchez for their help during data acquisition.

\section{Funding}

This work was supported by internal funds.

\section{Availability of data and materials}

The dataset supporting the conclusions of this article is available in the Figshare repository https://dx.doi.org/10.6084/m9.figshare.3380845.v1.

\section{Authors' contributions}

PTG and MECH conducted the analysis and drafted the manuscript. JSO, APdL, and LGG designed the study and drafted the manuscript. MBdV and AMG conducted microbiologic tests and drafted the manuscript. LPCH conducted the analysis. All authors read and approved the final manuscript.

\section{Competing interests}

The authors declare that they have no competing interests.

\section{Consent for publication}

Not applicable.

\section{Ethics approval and consent to participate}

The study was conducted in accordance with the ethical standards of the Helsinki Declaration. The Ethics Commission of Instituto Nacional de Ciencias Medicas y Nutricion Salvador Zubiran approved the study and permitted the use of information contained in the patients' records given the study's retrospective nature and after all individual person's identifiers were removed for analysis.

\section{Author details}

'Department of Infectious Diseases, Laboratory of Clinical Microbiology, Instituto Nacional de Ciencias Médicas y Nutrición Salvador Zubirán, Mexico City, Mexico. ${ }^{2}$ Centro de Investigación sobre Enfermedades Infecciosas, Instituto Nacional de Salud Pública, Cuernavaca, Morelos, Mexico.

${ }^{3}$ Department of Medicine, Instituto Nacional de Ciencias Médicas y Nutrición 
Salvador Zubirán, Av. Vasco de Quiroga \#15, Tlalpan, Belisario Domínguez Sección XVI, 14080 Mexico City, Mexico.

Received: 20 May 2016 Accepted: 28 October 2016 Published online: 08 November 2016

\section{References}

1. Cosivi O, Grange JM, Daborn CJ, Raviglione MC, Fujikura T, Cousins D, Robinson RA, Huchzermeyer HF, de Kantor I, Meslin FX. Zoonotic tuberculosis due to Mycobacterium bovis in developing countries. Emerg Infect Dis. 1998:4:59-70.

2. de la Rua-Domenech R. Human Mycobacterium bovis infection in the United Kingdom: Incidence, risks, control measures and review of the zoonotic aspects of bovine tuberculosis. Tuberculosis (Edinb). 2006:86:77-109

3. Bobadilla-del Valle M, Torres-Gonzalez P, Cervera-Hernandez ME, MartinezGamboa A, Crabtree-Ramirez B, Chavez-Mazari B, Ortiz-Conchi N, RodriguezCruz L, Cervantes-Sanchez A, Gudino-Enriquez T, et al. Trends of mycobacterium bovis isolation and first-line anti-tuberculosis drug susceptibility profile: a fifteen-year laboratory-based surveillance. PLoS Neg Trop Dis. 2015;9:e0004124.

4. Grange JM. Mycobacterium bovis infection in human beings. Tuberculosis (Edinb). 2001;81:71-7.

5. Konno K, Feldmann FM, McDermott W. Pyrazinamide susceptibility and amidase activity of tubercle bacilli. Am Rev Respir Dis. 1967;95:461-9.

6. LoBue PA, Moser KS. Treatment of Mycobacterium bovis infected tuberculosis patients: San Diego County, California, United States, 1994-2003. Int J Tuberc Lung Dis. 2005;9:333-8.

7. LoBue PA, LeClair JJ, Moser KS. Contact investigation for cases of pulmonary Mycobacterium bovis. Int J Tuberc Lung Dis. 2004;8:868-72.

8. Guerrero A, Cobo J, Fortun J, Navas E, Quereda C, Asensio A, Canon J, Blazquez J, Gomez-Mampaso E. Nosocomial transmission of Mycobacterium bovis resistant to 11 drugs in people with advanced HIV-1 infection. Lancet. 1997;350:1738-42.

9. Evans JT, Smith EG, Banerjee A, Smith RM, Dale J, Innes JA, Hunt D, Tweddell A, Wood A, Anderson C, et al. Cluster of human tuberculosis caused by Mycobacterium bovis: evidence for person-to-person transmission in the UK. Lancet. 2007;369:1270-6.

10. Hlavsa MC, Moonan PK, Cowan LS, Navin TR, Kammerer JS, Morlock GP, Crawford JT, Lobue PA. Human tuberculosis due to Mycobacterium bovis in the United States, 1995-2005. Clin Infect Dis. 2008:47:168-75.

11. Gallivan M, Shah N, Flood J. Epidemiology of human Mycobacterium bovis disease, California, USA, 2003-2011. Emerg Infect Dis. 2015;21:435-43.

12. Majoor CJ, Magis-Escurra C, van Ingen J, Boeree MJ, van Soolingen D. Epidemiology of Mycobacterium bovis disease in humans, The Netherlands, 1993-2007. Emerg Infect Dis. 2011;17:457-63.

13. LoBue PA, Betacourt W, Peter C, Moser KS. Epidemiology of Mycobacterium bovis disease in San Diego County, 1994-2000. Int J Tuberc Lung Dis. 2003; 7:180-5.

14. Park D, Qin H, Jain S, Preziosi M, Minuto JJ, Mathews WC, Moser KS, Benson CA. Tuberculosis due to Mycobacterium bovis in patients coinfected with human immunodeficiency virus. Clin Infect Dis. 2010;51:1343-6.

15. Rodwell TC, Moore M, Moser KS, Brodine SK, Strathdee SA. Tuberculosis from Mycobacterium bovis in binational communities, United States. Emerg Infect Dis. 2008;14:909-16.

16. Baez-Saldana R, Villafuerte-Garcia A, Cruz-Hervert P, Delgado-Sanchez G, Ferreyra-Reyes L, Ferreira-Guerrero E, Mongua-Rodriguez N, MonteroCampos R, Melchor-Romero A, Garcia-Garcia L. Association between highly active antiretroviral therapy and type of infectious respiratory disease and All-cause in-hospital mortality in patients with HIV/AIDS: a case series. PLoS One. 2015;10:e0138115.

17. SENASICA. Situación actual. 2016. http://www.senasica.gob.mx/?id=4369. Accessed 1 Jan 2016.

18. Centers for Disease Control and Prevention. Targeted tuberculin testing and treatment of latent tuberculosis infection. American Thoracic Society. MMWR Recomm Rep. 2000;49:1-51.

19. Vestal AL. Procedures for the isolation and identification of mycobacteria. 1st ed. Washington: United States Government Printing Office; 1969.

20. Lennette EH, Spaulding EH, Truant JP. Manual of clinical microbiology. 2dth ed. Washington: American Society for Microbiology; 1974.
21. Balows A. Manual of clinical microbiology. 5th ed. Washington, D.C. American Society for Microbiology; 1991.

22. Kamerbeek J, Schouls L, Kolk A, van Agterveld M, van Soolingen D, Kuijper S, Bunschoten A, Molhuizen H, Shaw R, Goyal M, van Embden J. Simultaneous detection and strain differentiation of Mycobacterium tuberculosis for diagnosis and epidemiology. J Clin Microbiol. 1997;35:907-14.

23. Richter E, Weizenegger M, Fahr AM, Rusch-Gerdes S. Usefulness of the GenoType MTBC assay for differentiating species of the Mycobacterium tuberculosis complex in cultures obtained from clinical specimens. J Clin Microbiol. 2004;42:4303-6.

24. Jonsson J, Hoffner S, Berggren I, Bruchfeld J, Ghebremichael S, Pennhag A, Groenheit R. Comparison between RFLP and MIRU-VNTR genotyping of Mycobacterium tuberculosis strains isolated in Stockholm 2009 to 2011. PLoS One. 2014;9:e95159.

25. Cordova E, Gonzalo X, Boschi A, Lossa M, Robles M, Poggi S, Ambroggi M. Human Mycobacterium bovis infection in Buenos Aires: epidemiology, microbiology and clinical presentation. Int J Tuberc Lung Dis. 2012;16(3):415-7.

26. Lopez-Alvarez R, Badillo-Lopez C, Cerna-Cortes JF, Castillo-Ramirez I, RiveraGutierrez S, Helguera-Repetto AC, Aguilar D, Hernandez-Pando R, Samper S, Gonzalez-y-Merchand JA. First insights into the genetic diversity of Mycobacterium tuberculosis isolates from HIV-infected Mexican patients and mutations causing multidrug resistance. BMC Microbiol. 2010;10:82.

27. Pereira-Suárez AL, Estrada-Chávez Y, Zúñiga-Estrada A, Lopez-Rincón G, Hernández DU, Padilla-Ramírez FJ, Estrada-Chávez C. Detection of Mycobacterium tuberculosis complex by PCR in fresh cheese from local markets in Hidalgo. Mexico J Food Prot. 2014;77(5):849-52.

28. Grange JM, Daborn C, Cosivi O. HIV-related tuberculosis due to Mycobacterium bovis. Eur Respir J. 1994;7:1564-6.

29. Jick SS, Lieberman ES, Rahman MU, Choi HK. Glucocorticoid use, other associated factors, and the risk of tuberculosis. Arthritis Rheum. 2006;55:19-26.

30. O'Garra A, Redford PS, McNab FW, Bloom Cl, Wilkinson RJ, Berry MP. The immune response in tuberculosis. Annu Rev Immunol. 2013;31:475-527.

31. World Health Organization.Tuberculosis in the Americas: Regional Report 2014. Epidemiology, Control and Financing. 2014 . http://www.paho.org/hq/ index.php?option=com_docman\&task=doc_view\&ltemid=270\&gid= 31284\&lang=es. Accessed 25 Aug 2016

32. Torres-Gonzalez P, Soberanis-Ramos O, Martinez-Gamboa A, Chavez-Mazari B, Barrios-Herrera MT, Torres-Rojas M, Cruz-Hervert LP, Garcia-Garcia L, Singh M, Gonzalez-Aguirre A, et al. Prevalence of latent and active tuberculosis among dairy farm workers exposed to cattle infected by Mycobacterium bovis. PLoS Negl Trop Dis. 2013;7:e2177.

33. Lonnroth $\mathrm{K}$, Jaramillo E, Williams BG, Dye C, Raviglione M. Drivers of tuberculosis epidemics: the role of risk factors and social determinants. Soc Sci Med. 2009;68:2240-6.

34. Berg S, Smith NH. Why doesn't bovine tuberculosis transmit between humans? Trends Microbiol. 2014;22:552-3.

35. Gambhir HS, Kaushik RM, Kaushik R, Sindhwani G. Tobacco smoking-associated risk for tuberculosis: a case-control study. Int Health. 2010;2:216-22.

36. Bonacci RA, Cruz-Hervert LP, Garcia-Garcia L, Reynales-Shigematsu LM, Ferreyra-Reyes L, Bobadilla-del-Valle M, Canizales-Quintero S, FerreiraGuerrero E, Baez-Saldana R, Tellez-Vazquez N, et al. Impact of cigarette smoking on rates and clinical prognosis of pulmonary tuberculosis in Southern Mexico. J Infect. 2013;66:303-12.

37. Bates MN, Khalakdina A, Pai M, Chang L, Lessa F, Smith KR. Risk of tuberculosis from exposure to tobacco smoke: a systematic review and meta-analysis. Arch Intern Med. 2007;167:335-42.

38. Chuang HC, Su CL, Liu HC, Feng PH, Lee KY, Chuang KJ, Lee CN, Bien MY. Cigarette smoke is a risk factor for severity and treatment outcome in patients with culture-positive tuberculosis. Ther Clin Risk Manag. 2015;11:1539-44.

39. Kurbatova EV, Cavanaugh JS, Dalton T, Click ES, Cegielski JP. Epidemiology of pyrazinamide-resistant tuberculosis in the United States, 1999-2009. Clin Infect Dis. 2014;57:1081-93.

40. Centers for Disease Control and Prevention. Human tuberculosis caused by Mycobacterium bovis-New York City, 2001-2004. Morb Mortal Wkly Rep. 2005:54:605-8. 